\title{
Caracterização morfométrica de bacias hidrográficas através de dados SRTM
}

\author{
Paulo T. S. de Oliveira ${ }^{1}$, Teodorico Alves Sobrinho ${ }^{1}$, Jorge L. Steffen ${ }^{1} \&$ Dulce B. B. Rodrigues ${ }^{1}$
}

\section{RESU MO}

Visa-se, neste trabalho, avaliar os dados Shuttle Radar Topography Mission (SRTM) na caracterização morfométrica de bacias hidrográficas, através da comparação das características obtidas a partir de dados SRTM e de cartas topográficas, processados em Sistema de Informação Geográfica (SIG). 0 estudo foi realizado tomando-se por base a bacia hidrográfica do Ribeirão Salobra, com área aproximada de $540 \mathrm{~km}^{2}$. A diferença percentual obtida nos dados morfométricos entre os métodos estudados foi inferior a $11 \%$, exceto no índice de circularidade (22\%) e declividade média (55\%). A utilização de dados SRTM em ambiente SIG permite a caracterização morfométrica de bacias hidrográficas, podendo auxiliar a gestão e o gerenciamento dos recursos hídricos, mostrando-se uma alternativa prática e viável ao minimizar custos e tempo na execução dos trabalhos.

Palavras-chave: modelo digital de elevação, gestão de recursos hídricos, hidrologia

\section{Morphometric characterization of watershed through SRTM data}

\begin{abstract}
This study aimed to evaluate the Shuttle Radar Topography Mission (SRTM) data in morphometric characterization of watersheds, through the comparison of the characteristics obtained from SRTM data and topographic letters, by using the Geographic Information System (GIS). The study considered the Ribeirão Salobra basin, its area is about $540 \mathrm{~km}^{2}$. The percentage difference in the morphometric data obtained between the two methods was less than 11\%, except for the index of circularity (22\%) and average slope (55\%). The use of SRTM data allows the morphometric characterization of watershed and can help management of water resources. In addition, this is an easy and economic alternative to minimize costs and job times.
\end{abstract}

Key words: digital elevation models, water resource management, hydrology 


\section{INTRODUÇÃO}

No desenvolvimento de estudos ambientais é fundamental a análise das características morfométricas de bacias hidrográficas. De acordo com Tonello et al. (2006), as características físicas e bióticas de uma bacia exercem importante papel nos processos do ciclo hidrológico influenciando, dentre outros, a infiltração, a quantidade de água produzida como deflúvio, a evapotranspiração e o escoamento superficial e subsuperficial.

Atualmente, a caracterização morfométrica de bacias hidrográficas é feita com a integração de informações de relevo em ambiente de Sistema de Informações Geográficas (SIG). Este procedimento pode ser realizado de modo manual ou automático (Cardoso et al., 2006). As informações de relevo são representadas por uma estrutura numérica de dados correspondente à distribuição espacial da altitude e da superfície do terreno, denominada modelo digital de elevação (MDE). Esses modelos são obtidos, hoje, por meio da interpolação de curvas de nível extraídas de uma carta topográfica ou através de imagens de sensores remotos.

A utilização de MDE em SIG apresenta vantagens, como os recursos digitais (velocidade, repetibilidade e integração com outras bases de dados), a redução de intervenções manuais e, portanto, subjetividade e a possibilidade de representação paramétrica (Valeriano et al., 2006). Assim, os MDE vêm sendo utilizados em estudos de recursos hídricos, como no delineamento de redes de drenagem, limites de bacias hidrográficas, cálculo de declividade, altitude, na verificação da direção de fluxo do escoamento superficial e como parte integrante de modelos hidrológicos (Mark, 1984; Band, 1986; Jenson \& Domingue, 1988; Fairfield \& Leymarie, 1991; Verdin \& Verdin, 1999; Valeriano, 2003; Ribeiro et al., 2008).

MDE's produzidos a partir de informações de sensoriamento remoto, tal como Iterferometric Synthetic Aperture Radar (InSAR), têm ganhado relevância por advirem de uma técnica rápida e acurada de coletar dados topográficos (Rabus et al., 2003). A missão Shuttle Radar Topography Mission (SRTM), realizada em 2000 a bordo da nave Endeavour, incorpora a técnica InSAR e dispõe publicamente seus dados em escala global por intermédio da United States Geological Survey (USGS). Assim, diversas pesquisas vêm sendo conduzidas objetivando-se analisar, comparar e atualizar informações da superfície terrestre por meio de dados SRTM (Valeriano et al., 2006; Ludwig \& Schneider, 2006; Fredrick et al., 2007; Rennó et al., 2008; Alcaraz et al., 2009).

Gerstenecker et al. (2005) avaliando várias bases para geração de MDE, inclusive cartas topográficas, concluíram que a missão SRTM é um passo de considerável importância no detalhamento acurado dos MDE's do globo terrestre. Santos et al. (2006) notaram que o MDE obtido a partir de dados SRTM apresentou melhores resultados altimétricos quando comparado com o MDE gerado a partir de cartas topográficas escalas 1:100.000. Oliveira \& Paradella (2008) estudando uma área de relevo plano e montanhoso da região Amazônica, concluíram que o MDE derivado do SRTM atendeu aos padrões de exatidão cartográficos para a escala de 1:100.000 Classe A.
Assim, este trabalho foi realizado com o objetivo de avaliar a caracterização morfométrica de bacias hidrográficas através de dados do projeto SRTM processados em SIG, comparando-os com resultados obtidos a partir de cartas topográficas.

\section{MATERIAL E MÉTODOS}

\section{Área em estudo}

O estudo foi realizado tomando-se por base a bacia hidrográfica do Ribeirão Salobra, com área aproximada de $540 \mathrm{~km}^{2}$, inserida no Município de Terenos, região central do Estado de Mato Grosso do Sul (Figura 1). Esta bacia está localizada em um relevo de transição entre a escarpa da Serra de Maracaju e a depressão pantaneira.

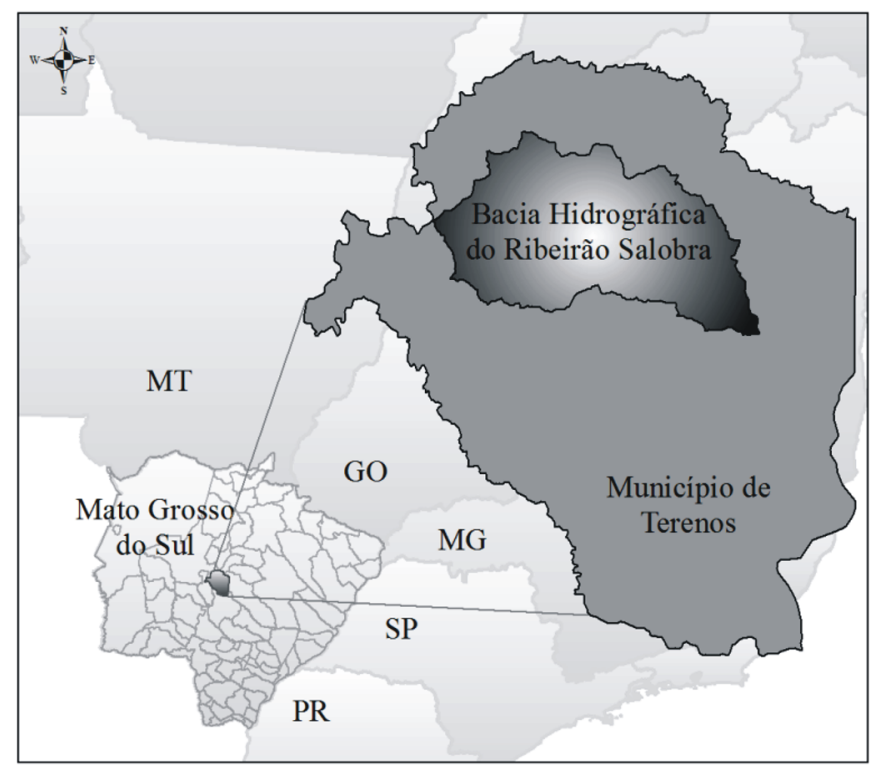

Figura 1. Localização da área em estudo

\section{Processamento dos dados SRTM}

Obtiveram-se os dados SRTM junto à USGS, com informações originais disponíveis para a América do Sul referentes à banda $\mathrm{C}$ do equipamento InSAR, apresentando resolução espacial de $90 \mathrm{~m}$ e elipsóide de referência WGS84. O processo de caracterização morfométrica da bacia hidrográfica foi desenvolvido no SIG ArcGIS 9.2 (ESRI, 2006), juntamente com as extensões (plugins) Spatial Analyst e Hydrology Modeling.

A metodologia utilizada no processamento dos dados SRTM foi subdivida em quatro etapas, sendo: preenchimento de sinks (fill sinks); direção de fluxo (flow direction), fluxo acumulado (flow accumulation) e delimitação de bacias (Watershed).

A acurácia dos dados SRTM tem sido comprovada por vários pesquisadores (Kocak et al., 2005; Rodriguez et al., 2006); no entanto, estudos desenvolvidos por Falorni et al. (2005) e Kääb (2005) apontam que a acurácia dos dados SRTM depende da topografia local, constatando-se maior 
incidência de erros em dados referentes a terrenos com relevo íngreme ou montanhoso. De acordo com Luedeling et al. (2007), o MDE que acompanha os dados SRTM contém falhas em certas áreas do globo, originadas principalmente de duas maneiras: da ocorrência de corpos hídricos e do relevo acidentado; nesta última, a frequência de falhas é maior em superfícies com inclinação acima de $20^{\circ}$, devido ao sombreamento ocasionado no radar.

As falhas no MDE advindas dos dados SRTM são denominadas sinks que, de acordo com Mendes \& Cirilo (2001) se caracterizam por áreas rodeadas de elevações com valores de cotas superiores, semelhante a uma depressão. O preenchimento dessas pequenas depressões é o primeiro tratamento dado à matriz de altitudes. Essas depressões ou sinks, são consideradas empecilhos ao escoamento durante a aplicação de modelos hidrológicos, sedimentológicos e de poluentes de origem difusa. Realizaram-se as correções no MDE por meio da função fill sinks, que considera as altitudes dos pixels vizinhos, de forma a preencher os sinks promovendo, assim, a geração do MDE com melhor consistência.

A direção de fluxo define as relações hidrológicas entre pontos diferentes dentro de uma bacia hidrográfica. Consequentemente, a continuidade topológica para as direções de fluxo é necessária para que uma drenagem funcional possa existir. As conexões hidrológicas de direção de fluxo entre dois pontos em uma superfície não são as mesmas que aquelas baseadas em distância Euclidiana (Rennó et al., 2008). Obteve-se a direção de fluxo de água na rede de drenagem pela função flow direction, que gera uma grade regular definindo as direções de fluxo, tomando-se por base a linha de maior declividade do terreno. A nova grade numérica gerada determina a direção de maior declividade de um pixel em relação aos seus oito pixels vizinhos; desta forma, ocorre a descrição numérica da direção que a água irá percorrer após atingir cada pixel.

A partir da grade regular gerada de direção de fluxo, o fluxo acumulado foi obtido pela função flow accumulation. De acordo com Mendes \& Cirilo (2001) o fluxo acumulado representa a rede hidrográfica, sendo possível montar uma nova grade contendo os valores de acúmulo de água em cada pixel. Deste modo, cada pixel recebe um valor correspondente ao número de pixels que contribuem para que a água chegue até ele. A partir da direção de fluxo, o fluxo acumulado é obtido somando-se a área das células (quantidade de células) na direção do fluxo (ou escoamento).

A delimitação de bacias é realizada processando-se os mapas de direção de fluxo e fluxo acumulado na função Watershed. O valor de área de cada bacia hidrográfica a ser gerada corresponde à quantidade de células que são processadas. Como cada célula do MDE obtido da SRTM possui pixels de $90 \mathrm{~m}$, a área de cada pixel é equivalente a $8.100 \mathrm{~m}^{2}$. Deste modo, realizaram-se testes até se obter a definição do valor de células que melhor correspondesse à área de estudo, sendo este de 45.000 células, processando bacias de até $364.500 .000 \mathrm{~m}^{2}$, equivalentes a 36.450 ha.

Depois de se processar as informações na função Watershed, gerou-se a delimitação das bacias que, posteriormente, foram convertidas para o formato vetorial, na função
Raster to Features da extensão Spatial Analyst; após a delimitação das bacias hidrográficas utilizou-se o comando Stream Network para delimitação da hidrografia, tanto para os resultados da direção de fluxo como para fluxo acumulado, adotando-se valor 500 como o número mínimo de células para a geração de fluxo.

Obtiveram-se, ainda, resultados de altitude, declividade e informações para compor a caracterização morfométrica da bacia, tais como: comprimento da bacia, comprimento do rio principal e ordem dos cursos d'água.

\section{Processamento dos dados obtidos de cartas topográficas}

Utilizaram-se as cartas topográficas folhas Campo Grande SF. 21-X-B-II (DSG, 1979) e Palmeiras SF. 21-X-B-III (DSG, 1988), ambas na escala 1:100.000, representadas por curvas de nível equidistantes $40 \mathrm{~m}$, pontos cotados e hidrografia. Realizou-se a digitalização das cartas com resolução de 300 dpi e, posteriormente, o georreferenciamento tomando-se por base coordenadas planas, elipsóide de referência Córrego Alegre, Fuso $21 \mathrm{~S}$, processo este desenvolvido no SIG SPRING 5.0.2 (Câmara et al., 1996). O georreferenciamento foi realizado através de polinômio de $2^{\circ}$ grau e do interpolador vizinho mais próximo. As imagens foram registradas com 20 pontos de controle obtendo-se valores de erro inferiores a 0,5 pixel.

Realizou-se o processo de vetorialização dos atributos das cartas topográficas no SIG ArcGis 9.2, geração do MDE de resolução 30 x $30 \mathrm{~m}$ e, a partir deste, a declividade da área em estudo. Utilizando-se os atributos vetorializados obtiveram-se, de modo manual, a delimitação da bacia hidrográfica (área, perímetro e comprimento), o comprimento do rio principal e a ordem dos cursos d'água.

\section{Morfometria da bacia hidrográfica}

De posse dos resultados obtidos por meio dos dados SRTM e das cartas topográficas, diferentes características físicas da bacia hidrográfica em estudo foram comparadas, tais como: área da bacia; perímetro; coeficiente de compacidade; fator de forma; índice de circularidade; declividade; altitude; densidade de drenagem e ordem dos cursos d'água.

O coeficiente de compacidade (Kc) obtido pela Eq. 1, relaciona a forma da bacia com um círculo e constitui a relação entre o perímetro da bacia e a circunferência de um círculo de área igual à da bacia (Cardoso et al., 2006).

$$
\mathrm{Kc}=0,28 \frac{\mathrm{P}}{\sqrt{\mathrm{A}}}
$$

em que:

$$
\begin{gathered}
\text { Kc - coeficiente de compacidade } \\
\mathrm{P} \text { - perímetro da bacia, } \mathrm{m} \\
\mathrm{A} \text { - área de drenagem, } \mathrm{m}^{2}
\end{gathered}
$$

Bacias hidrográficas cuja forma se aproxima à de um circulo, tendem a proporcionar a conversão do escoamento superficial para um trecho pequeno do rio principal; assim, quanto mais próximo a 1 for este índice maior a potencialidade de picos de enchentes na bacia hidrográfica.

$\mathrm{O}$ fator de forma $(\mathrm{F})$ relaciona a forma da bacia com a de 
um retângulo, correspondendo à razão entre a largura média e o comprimento axial da bacia (Eq. 2).

$$
\mathrm{F}=\frac{\mathrm{A}}{\mathrm{L}^{2}}
$$

em que:

$$
\begin{aligned}
& \mathrm{F} \text { - fator de forma } \\
& \mathrm{A} \text { - área de drenagem, } \mathrm{m}^{2} \\
& \mathrm{~L} \text { - comprimento do eixo da bacia, } \mathrm{m}
\end{aligned}
$$

Uma bacia com $\mathrm{F}$ baixo possui menor propensão a enchentes que outra com a mesma área mas com F maior, em virtude de que em uma bacia estreita e longa ( $\mathrm{F}$ baixo), ocorre menor possibilidade de ocorrência de chuvas intensas cobrindo, simultaneamente, toda a sua extensão.

Segundo Cardoso et al. (2006), simultaneamente ao Kc, o índice de circularidade (IC) tende para a unidade à medida em que a bacia se aproxima da forma circular e diminui sempre que a forma se torna alongada. No cálculo do IC utilizou-se a Eq. 3.

$$
\mathrm{IC}=\frac{12,57 * \mathrm{~A}}{\mathrm{P}^{2}}
$$

em que:

$$
\begin{aligned}
& \text { IC - índice de circularidade } \\
& \mathrm{A} \text { - área de drenagem, } \mathrm{m}^{2} \\
& \mathrm{P} \text { - perímetro, } \mathrm{m}
\end{aligned}
$$

A rede hidrográfica foi ordenada de acordo com Strahler (1957), que considera todos os canais sem tributários como de primeira ordem; os canais de segunda ordem, por outro lado, se originam da confluência de dois canais de primeira ordem; os de terceira ordem, por sua vez, se formam pela junção de canais de ordem 2 .

A declividade do terreno consiste entre variação de altitude entre dois pontos do terreno e a distância horizontal que os separa. Na obtenção dos dados de declividade utilizaramse os MDE gerados a partir das diferentes base de dados (SRTM e cartas topográficas) e se aplicou a função slope disponível na extensão Spatial Analyst do ArcGIS 9.2.

A densidade de drenagem $\left(\mathrm{D}_{\mathrm{d}}\right)$ indica o nível de desenvolvimento do sistema de drenagem de uma bacia hidrográfica, fornecendo uma indicação da sua eficiência. $O$ cálculo de $D_{d}$ é expresso pela relação entre o somatório do comprimento total dos canais com a área da bacia de drenagem (Eq. 4).

$$
\mathrm{Dd}=\frac{\mathrm{Lt}}{\mathrm{A}}
$$

em que:

$$
\begin{aligned}
& \mathrm{D}_{\mathrm{d}} \text { - densidade de drenagem, } \mathrm{km} \mathrm{km}^{-2} \\
& \mathrm{~L}_{\mathrm{t}} \text { - comprimento total de todos os canais, } \mathrm{km} \\
& \mathrm{A} \text { - área de drenagem, } \mathrm{km}^{2}
\end{aligned}
$$

\section{RESULTADOS E DISCUSSÃO}

Os resultados da caracterização morfométrica da bacia hidrográfica do Ribeirão Salobra se encontram apresentados na Tabela 1 .
Tabela 1. Resultados obtidos da caracterização morfométrica

\begin{tabular}{lccc}
\hline Característica Física & SRTM & Cartas & Variação (\%) \\
Área de drenagem $\left(\mathrm{km}^{2}\right)$ & 543,9084 & 540,5540 & 1 \\
Perímetro $(\mathrm{km})$ & 123,4846 & 111,4923 & 10 \\
Lt $(\mathrm{km})$ & 167,2913 & 184,8591 & 11 \\
L $(\mathrm{km})$ & 40,6368 & 40,6599 & 0 \\
KC & 1,4825 & 1,3427 & 9 \\
F & 0,3290 & 0,3273 & 1 \\
IC & 0,4484 & 0,5466 & 22 \\
Declividade média (\%) & 3,73 & 1,69 & 55 \\
Ordem da bacia & 3 & 4 & - \\
Dd $\left(\mathrm{km} \mathrm{km}^{-2}\right)$ & 0,3076 & 0,3419 & 11 \\
\hline
\end{tabular}

Considerando os resultados obtidos pode-se classificar a bacia hidrográfica do Ribeirão Salobra como de baixa suscetibilidade a enchentes em condições normais de precipitação, evento constatado em virtude do coeficiente de compacidade $(\mathrm{Kc})$ apresentar o valor afastado da unidade e o fator de forma (F) exibir um valor baixo. Deste modo, pode-se considerar que a bacia em estudo não possui forma circular e, sim, tendência alongada.

Outro índice que indica a forma da bacia é o de circularidade (IC). De acordo com Schumm (1956), valores maiores que 0,51 mostram que a bacia tende a ser mais circular favorecendo os processos de inundação (picos de cheias); já os valores menores que 0,51 sugerem que a bacia tende a ser mais alongada contribuindo para o processo de escoamento. A comparação desse índice entre as duas formas estudadas indica uma diferença de $22 \%$. Enquanto os resultados de IC obtidos com dados SRTM se encontram abaixo de 0,51, os derivados de cartas topográficas estão pouco acima deste valor; tal diferença está relacionada com o perímetro da bacia obtido a partir das diferentes formas de dados, pois na área da bacia, outro dado de entrada na obtenção de IC, os valores se apresentam próximos.

A densidade de drenagem $\left(\mathrm{D}_{\mathrm{d}}\right)$ da bacia em estudo é baixa, indicando área permeável e de relevo plano e suave; a $\mathrm{D}_{\mathrm{d}}$ varia diretamente com a extensão do escoamento superficial concluindo-se que a bacia possui baixa suscetibilidade a enchentes, em condições normais de precipitação; a variação da $D_{d}$, em relação aos métodos estudados, foi de $11 \%$, em virtude principalmente da rede hidrográfica, constatando-se uma diferença de $17 \mathrm{~km}$ entre a estimada pelo MDE derivado do SRTM e aquela obtida por meio das cartas topográficas. Tal diferença da rede hidrográfica também proporcionou a obtenção de ordens diferentes para a bacia estudada (Tabela 1).

A variação entre os resultados obtidos a partir das fontes de dados estudadas pode ser explicada pela diferença entre o detalhamento dos MDE. Na Figura 2 se encontram os MDE e os dados de declividade obtidos a partir das duas fontes de dados estudados.

Nota-se um grau de detalhamento maior nos dados SRTM do que os obtidos de cartas topográficas. Fornelos \& Neves (2007) obtiveram resultados semelhantes quando compararam produtos oriundos de SRTM e cartas topográficas, concluindo que esta diferença se deve ao maior detalhamento 
A.

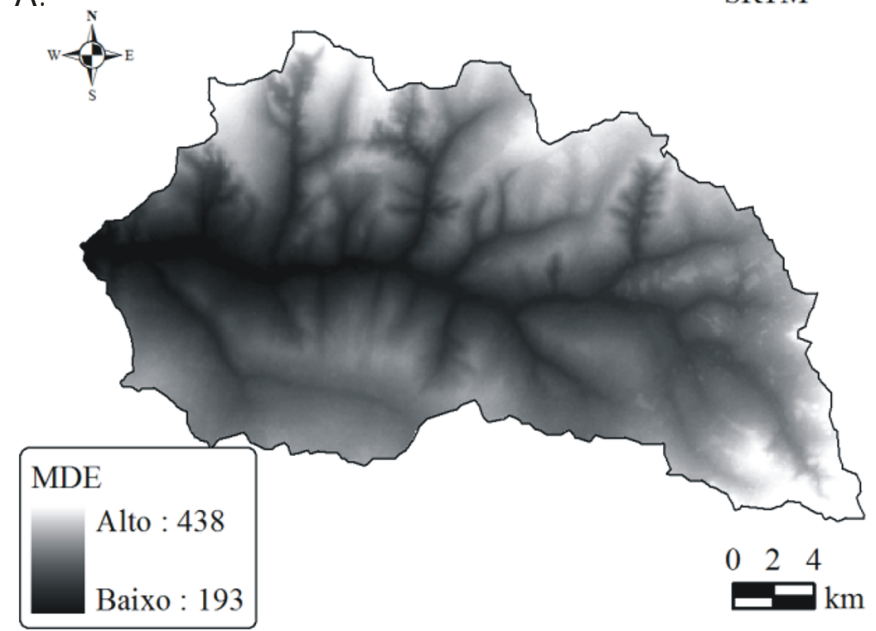

B.

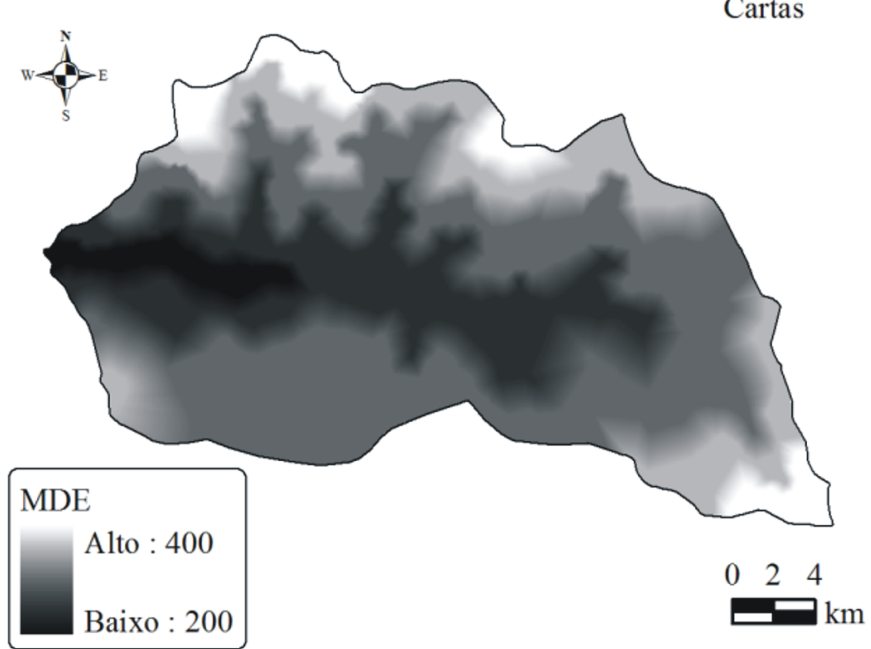

C.

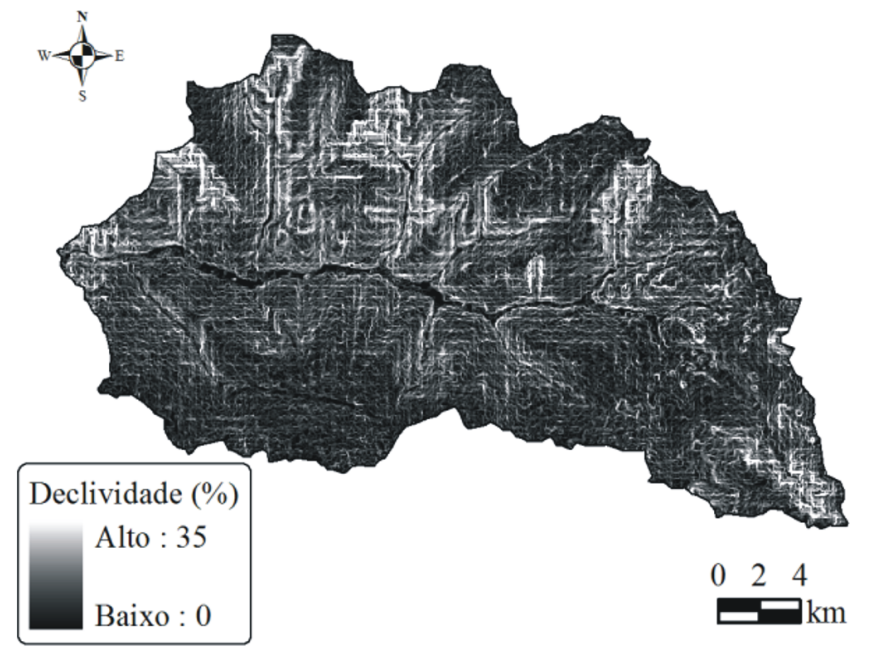

D.

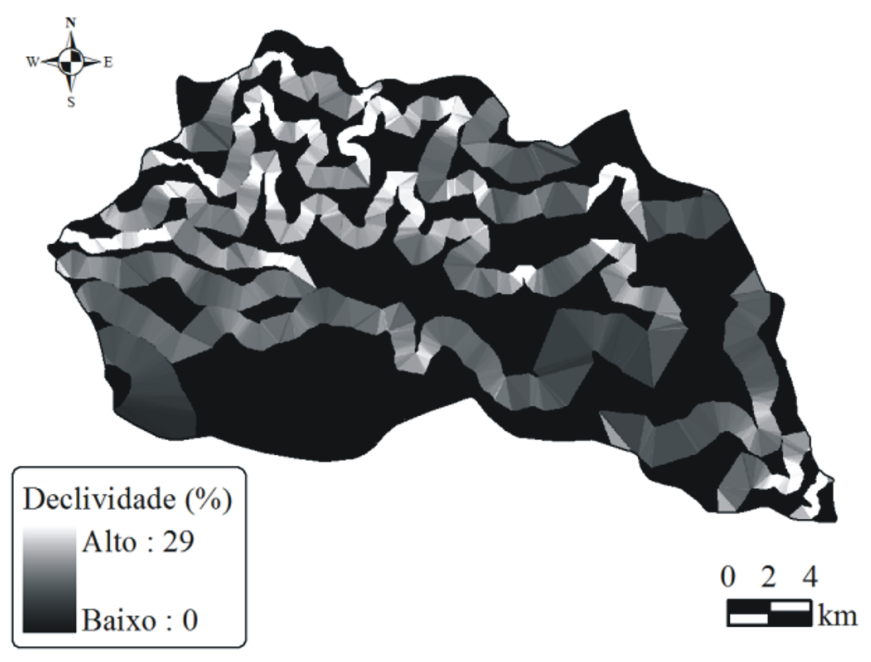

Figura 2. MDE e declividade da bacia do Ribeirão Salobra

em áreas planas. Comparando-se as Figuras 2C e 2D é possível verificar a afirmação dos autores, pois áreas consideradas de declividade nula na Figura 2D são representadas com detalhes de declive na Figura 2C.

O resultado obtido na delimitação da bacia hidrográfica com dados SRTM (Figura 3B) mostra-se compatível com o obtido por meio da delimitação manual a parir de cartas topográficas (Figura 3A). Assim, a utilização dos dados SRTM na delimitação de bacias hidrográficas pode ser considerada uma boa fonte de dados no desenvolvimento de estudos de recursos hídricos. Além disso, a realização de métodos de delimitação de bacias de modo automático apresenta menor subjetividade; deste modo, mesmo se realizando trabalhos em softwares SIG diferentes, os resultados serão mais próximos da realidade se comparados com os métodos manuais que variam com a percepção humana, conforme detalhado nos trabalhos de Jenson \& Domingue (1988) e Merkel et al. (2008).

Com relação ao método manual de obtenção dos dados, pode-se citar certa parcela de subjetividade, tanto na determinação das curvas de nível quanto da confecção do MDE. Nota-se, na obtenção dos dados de cartas topográficas, sua- vização das curvas de nível, o que não é observado nos dados obtidos de modo automático nos quais, de acordo Burrough \& MacDonnell (1998), as linhas e polígonos na estrutura raster se constituem de coordenadas ou pixels interligados que, quando transformados para estrutura de vetor, geram segmentos de retas que ligam pixels vizinhos. Essas diferenças resultam na maior variação entre os métodos estudados, sobretudo nas medidas lineares, como o perímetro da bacia e o comprimento total dos cursos d'água exercendo, assim, influência no índice de circularidade, densidade de drenagem e coeficiente de compacidade.

\section{CONCLUSÕES}

1. A utilização de dados SRTM em ambiente SIG permite a caracterização morfométrica de bacias hidrográficas podendo auxiliar a gestão e o gerenciamento dos recursos hídricos, razão por que se mostra uma alternativa prática e viável ao minimizar custos e tempo na execução dos trabalhos.

2. A variação da rede hidrográfica obtida pelos métodos 


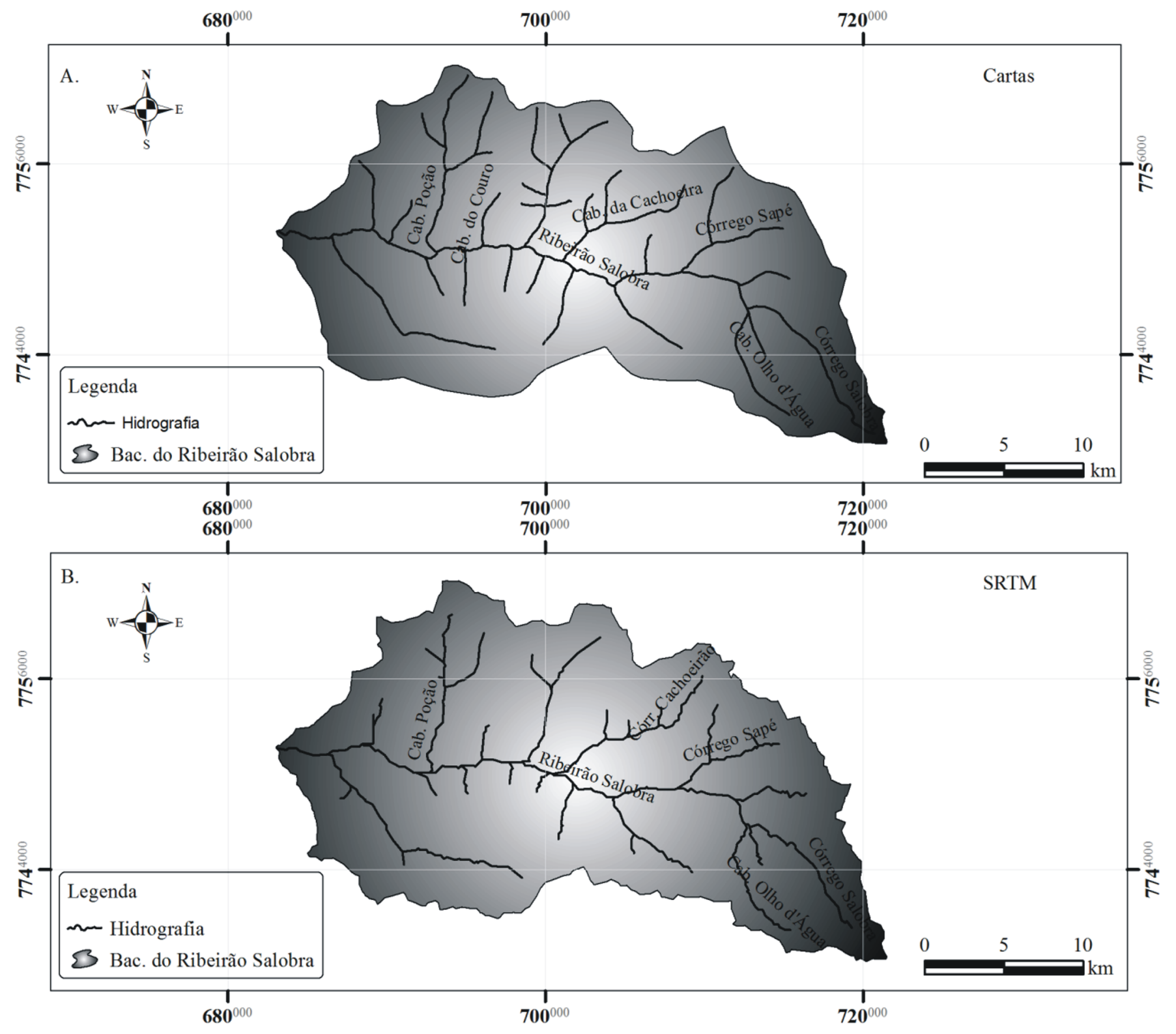

Figura 3. Delimitação da bacia hidrográfica do Ribeirão Salobra

estudados é de 17 km. Deste modo, na realização de outros estudos é recomendável a comparação da hidrografia obtida a partir de dados SRTM com outros dados de sensoriamento remoto ou cartas topográficas.

3. A delimitação automática de bacias hidrográficas por meio do processamento de dados SRTM em ambiente SIG, apresenta-se vantajosa em relação ao custo e benefício proporcionado, além de estabelecer a padronização do traçado e posterior minimização de conflitos quanto à fixação da unidade elementar de gestão dos recursos hídricos.

4. A partir dos resultados obtidos nos dois métodos estudados pode-se classificar a bacia hidrográfica do Ribeirão Salobra como de baixa suscetibilidade a enchentes em condições normais de precipitação.

\section{AGRADECIMENTOS}

Os autores expressam seus agradecimentos ao CNPq e à FUNDECT, pelo auxílio financeiro concedido para a realização do trabalho.

\section{LITERATURA CITADA}

Alcaraz, S. A.; Sannier, C.; Vitorino, A. C. T.; Daniel, O. Comparison of methodologies for automatic generation of limits and drainage networks for hidrographic basins. Revista Brasileira de Engenharia Agrícola e Ambiental, v.13, n.14, p.369-375, 2009. 
Band, L. E. Topographic partition of watershed with digital elevation models. Water Resource Research, v.22, n.1, p.15-24, 1986.

Burrough, P. A.; Macdonnell. Principles of geographical information systems. New York: Oxfor University Press, 1998. 333p.

Câmara, G.; Souza, R. C. M.; Freitas, U. M.; Garrido, J. SPRING: Integrating remote sensing and GIS by object-oriented data modelling. Computers \& Graphics, v.20, n.3, p.395-403, 1996.

Cardoso, C. A.; Dias, H. C. T.; Soares, C. P. B.; Martins, S. V. Caracterização morfométrica da Bacia Hidrográfica do Rio Debossan, Nova Friburgo, RJ. Revista Árvore, v.30, n.2, p.241-248, 2006.

DSG - Diretoria do Serviço Geográfico Brasileiro. Carta Campo Grande. Folha SF. 21-X-B-II. Escala 1:100.000. Ministério do Exército. Região Centro-Oeste do Brasil. Carta, 1979.

DSG - Diretoria do Serviço Geográfico Brasileiro. Carta Palmeira. Folha SF. 21-X-B. III. Escala 1:100.000. Ministério do Exército. Região Centro-Oeste do Brasil. Carta, 1988.

ESRI - Environmental Systems Research Institute. Inc. ArcGIS Professional GIS for the desktop, version 9.2. Software. 2006.

Fairfield, J.; Leymarie, P. Drainage networks from grid digital elevation models. Water Resources Research, v.27, n.5, p.709-717, 1991.

Falorni, G.; Teles, V.; Vivoni, E. R.; Bras, R. L. Amaratunga, K. S. Analysis and characterization of the vertical accuracy of digital elevation models from the Shuttle Radar Topography Mission. Journal of Geophysical Research, v.110, p.1-20, 2005.

Fornelos, L. F.; Neves, S. M. A. S. Uso de modelos digitais de elevação (MDE) gerados a partir de imagens de radar interferométricos (SRTM) na estimativa de perdas de solo. Revista Brasileira de Cartografia, v.59, n.1. p.25-33, 2007.

Fredrick, K. C.; Becker, M. W.; Matott, L. S.; Daw, A.; Bandilla, K.; Flewelling, D. M. Development of a numerical groundwater flow model using SRTM elevations. Hydrogeology Journal, v.15, p.171-181, 2007.

Gerstenecker, C.; Läufer, G. L.; Steineck, D.; Tiede, C.; Wrobel, B. Validation of digital elevation models around Merapi Volcano, Java, Indonesia. Natural Hazards and Earth System Sciences, v.5, p.863-876, 2005.

Jenson, S. K.; Domingue, J. O. Extracting topographic structure from digital elevation data for geographic information system analysis. Photogrammetric Engineering and Remote Sensing, v.54, n.11, p.1593-1600, 1988.

Kääb, A. Combination of SRTM3 and repeat ASTER data for deriving alpine glacier flow velocities in the Bhutan Himalaya. Remote Sensing of Environment, v.94, n.4, p.463-474, 2005.

Kocak, G.; Buyuksalih, G.; Oruc, M. Accuracy assessment of interferometric digital elevation models derived from the Shuttle Radar Topography Mission X- and C-band data in a test area with rolling topography and moderate forest cover. Optical Engineering, v.44, n.3, p.036201-1-7, 2005.

Ludwig, R.; Schneider, P. Validation of digital elevation models from SRTM X-SAR for applications in hydrologic modeling. ISPRS Journal of Photogrammetry \& Remote Sensing, v.60, p.339-358, 2006.
Luedeling, E.; Siebert, S.; Buerkert, A. Filling the voids in the SRTM elevation model - A TIN-based delta surface approach. ISPRS Journal of Photogrammetry \& Remote Sensing, v.62, p.283-294, 2007.

Mark, D. M. Automatic detection of drainage networks from digital elevation models. Cartographica, v.21. p.168-178, 1984.

Mendes, C .A. B.; Cirilo, J. A. Geoprocessamento em recursos hídricos: Princípios, integração e aplicação. Porto Alegre: ABRH, 2001. 536p.

Merkel, W. H.; Kaushika, R. M.; Gorman, E. NRCS GeoHydro A GIS interface for hydrologic modeling. Computers \& Geosciences, v.34, p.918-930, 2008.

Oliveira, C. G.; Paradella, W. R. An assessment of the altimetric information derived from spaceborne SAR (RADARSAT-1, SRTM3) and optical (ASTER) data for cartographic application in the Amazon region. Sensors, v.8, p.3819-3829, 2008.

Rabus, B.; Eineder, M.; Roth, A.; Bamler, R. The shuttle radar topography mission: a new class of digital elevation models acquired by spaceborne radar. ISPRS Journal of Photogrammetry \& Remote Sensing, v.57, n.4. p.241-262, 2003.

Rennó, C. D.; Nobre, A. D.; Cuartas, L. A.; Soares, J. V.; Hodnett, M. G.; Tomasella, J.; Waterloo, M. J. HAND, a new terrain descriptor using SRTM-DEM: Mapping terra-firme rainforest environments in Amazonia. Remote Sensing of Environment, v.112, p.3469-3481, 2008.

Ribeiro, C. A. A. S.; Soares, V. P.; Santos, R. M.; Soares, C. P. B. Estruturação topológica de grandes bases de dados de bacias hidrográficas. Revista Árvore, v.32, n.4, p.687-696, 2008.

Rodriguez, E.; Morris, C. S.; Belz, J. E. A global assessment of the SRTM performance. Photogrammetric Engineering and Remote Sensing, v.72, n.3. p.249-260, 2006.

Santos, P. R. A.; Gaboardi, C.; Oliveira, L. C. Avaliação da precisão vertical dos modelos SRTM para a Amazônia. Revista Brasileira de Cartografia, v.58, n.1. p.101-107, 2006.

Schumm, S. A. Evolution of drainage systems and slopes in badlands of Perth Amboy. Geological Society of America Bulletin, v.67, n.5, p.597-646, 1956.

Strahler, A. N. Quantitative analysis of watershed geomorphology. Transaction of American Geophysical Union, v.38, p.913-920, 1957.

Tonello, K. C.; Dias, H. C. T.; Souza, A. L.; Alvares, C. A.; Ribeiro, S.; Leite, F. P. Morfometria da Bacia Hidrográfica da Cachoeira das Pombas, Guanhães - MG. Revista Árvore, v.30, n.5, p.849-857, 2006.

Valeriano, M. M. Mapeamento da declividade em microbacias com sistemas de informação geográfica. Revista Brasileira de Engenharia Agrícola e Ambiental, v.7, n.2, p.303-310, 2003.

Valeriano, M. M.; Kuplich, T. M.; Storino, M.; Amaral, B. D.; Mendes Júnior., J. N.; Lima, D. Modeling small watersheds in Brazilian Amazônia with SRTM-90m data. Computers \& Geosciences, v.32, n.8, p.1169-1181, 2006.

Verdin, K. L.; Verdin, J. P. A topological system for delineation and codification of the Earth's river basins. Journal of Hydrology, v.218, p.1-12, 1999. 